\title{
Genetic factors affecting patient responses to pancreatic cancer treatment
}

\author{
George Fotopoulos ${ }^{a}$, Konstantinos Syrigos ${ }^{\mathrm{a}, \mathrm{b}}$, Muhammad Wasif Saif ${ }^{\mathrm{c}}$ \\ University of Athens, Athens, Greece; Yale School of Medicine, New Haven, CT, USA; Tufts University School of \\ Medicine, Boston, Massachusetts, USA
}

\begin{abstract}
Cancer of the exocrine pancreas is a malignancy with a high lethal rate. Surgical resection is the only possible curative mode of treatment. Metastatic pancreatic cancer is incurable with modest results from the current treatment options. New genomic information could prove treatment efficacy. An independent review of PubMed and ScienceDirect databases was performed up to March 2016, using combinations of terms such pancreatic exocrine cancer, chemotherapy, genomic profile, pancreatic cancer pharmacogenomics, genomics, molecular pancreatic pathogenesis, and targeted therapy. Recent genetic studies have identified new markers and therapeutic targets. Our current knowledge of pancreatic cancer genetics must be further advanced to elucidate the molecular basis and pathogenesis of the disease, improve the accuracy of diagnosis, and guide tailor-made therapies.
\end{abstract}

Keywords Chemotherapy, genomics, molecular pancreatic pathogenesis, pancreatic exocrine cancer, targeted therapy

Ann Gastroenterol 2016; 29 (4): 1-11

\section{Introduction}

Cancer of the exocrine pancreas is a highly lethal malignancy, the fourth leading cause of cancer-related death in the United States, second only to colorectal cancer as a cause of digestive cancer-related death [1]. Surgical resection is the only possible curative mode of treatment. Unfortunately, this is the case only in about $15-20 \%$ of cases, as in the majority, patients present with advanced disease [1]. In this setting, whether locally advanced or metastatic, there are various treatment options such as chemoradiation and chemotherapy with various schemes, with mostly poor outcome.

A variety of drugs and combinations have been used in the adjuvant and metastatic setting. The most commonly used

${ }^{\mathrm{a}}$ Oncology Unit, Third Department of Medicine, University of Athens, Athens, Greece (George Fotopoulos, Konstantinos Syrigos); ${ }^{\text {'Yale }}$ School of Medicine, New Haven, CT, USA (Konstantinos Syrigos); 'Tufts University School of Medicine, Boston, Massachusetts, USA (Muhammad Wasif Saif)

\section{Conflict of Interest: None}

Correspondence to: George Fotopoulos, Oncology Unit, Third Department of Medicine, University of Athens, Athens, Greece, Sotiria Hospital, Mesogeion Avenue 152, 11527 Athens, Greece, Tel.: +30 2107700220 , e-mail: fotopoulos.george1@gmail.com

Received 9 March 2016; accepted 5 May 2016

published online 10 June 2016

DOI: http://dx.doi.org/10.20524/aog.2016.0056 agents include gemcitabine monotherapy [2,3] and gemcitabine combinations with erlotinib [4], oxaliplatin [5], cisplatin [6], capecitabine [7] and nab-paclitaxel [8]. Other agents used in various frequencies are capecitabine [9], fluouracil [3], eloxatin with capecitabine [10], and nab-paclitaxel [11]. In the adjuvant and locally advanced setting, the commonest practice is gemcitabine monotherapy [2], with or without radiation, and in the metastatic setting the standard of care is the use of gemcitabine with nab-paclitaxel or the scheme FOLFIRINOX $[11,12]$.

Not all patients fare the same with the same treatment. One of the main reasons for this, is the different molecular profile of the tumor and specific genetic factors affecting response to therapy. K-ras, hENT1, DCK, CDA, dihydropyrimidine dehydrogenase (DPD), HMLH1, TP1DPD, HER2, and SMAD4 are associated with management and response to pancreatic treatment (Table 1). We herein reviewed possible genetic factors affecting responses to therapy.

\section{Materials and methods}

An independent review of ScienceDirect and PubMed database was performed with a time period frame of five years up to March 2016, using combinations of terms such as pancreatic exocrine cancer, chemotherapy, genomic profile, pancreatic cancer pharmacogenomics, genomics, molecular pancreatic pathogenesis, and targeted therapy. No geographical restrictions were set and more than 500 articles were identified. 
Table 1 Genetic factors associated with management and response to pancreatic cancer treatment

\begin{tabular}{|c|c|c|}
\hline Targeted gene & Function & Significance in pancreatic cancer treatment \\
\hline KRAS & Cell cycle activation (MAPK and PIK3CA pathway) & $\begin{array}{l}\text { Constittutes anti-EGFR treatment ineffective. } \\
\text { Possible target of erlotinib }\end{array}$ \\
\hline hENT1 & Transports gemcitabine across the cellular membrane & Positive correlation with overall survival in gemcitabine therapy \\
\hline DCK & Phosphorylates gemcitabine to active form & Higher levels increase survival \\
\hline $\mathrm{CDA}$ & Metabolizes gemcitabine to its inactive form & Increased toxicity when inactive \\
\hline DPD & Metabolizes 5-FU & Low DPD levels associated with increased survival \\
\hline $\begin{array}{l}\text { hMLH1 } \\
\text { hMSH2 }\end{array}$ & Control the repair of DNA damage & $\begin{array}{l}\text { Pancreatic cancers with MSI also may be less responsive to } \\
\text { chemotherapeutic agents such as 5-FU }\end{array}$ \\
\hline TS & DNA and RNA synthesis & $\begin{array}{l}\text { Low levels of TS expression leads to a better response to capecitabine } \\
\text { and 5-FU }\end{array}$ \\
\hline SPARC & Regulates cell's interaction with its surrounding enviroment & mRNA SPARC expression is a prognostic factor \\
\hline HER1/EGFR & HER1/EGFR tyrosine kinase signaling & $\begin{array}{l}\text { Associated with poor prognosis and disease progression. } \\
\text { Blocking HER1/EGFR tyrosine kinase signaling decreases the growth } \\
\text { and metastasis of human pancreatic tumor xenografts } 16 \text { and improves } \\
\text { the anticancer effects of gemcitabine } \\
\text { Erlotinib is an oral HER1/EGFR tyrosine kinase inhibitor }\end{array}$ \\
\hline TP/DPD & Metabolizes capecitabine and catabolizes 5-FU & A survival benefit of 4 months correlated with lower TP/DPD ratio \\
\hline UGT1A1 & Metabolizes CPT-11 active form SN38 & $\begin{array}{l}\text { UGT1A1 activity exhibits a wide intersubject variability, in part related } \\
\text { to UGT1A1 gene polymorphisms }\end{array}$ \\
\hline HER2 & Widely expressed with low level & Potent target for anti-HER2 drugs \\
\hline BRCA2 & $\begin{array}{l}\text { Mutated in up to } 17 \% \text { of patients with familial pancreatic } \\
\text { cancer }\end{array}$ & Susceptible to DNA cross-linking agents and PARP inhibitors \\
\hline WOXX & SNP & Decreased expression interferes with gemcitabine sensitivity \\
\hline
\end{tabular}

The abstracts were screened to identify studies, and especially review articles, suitable for the purpose of this article. Identification and removal of duplicate articles was performed and the remaining articles were then reviewed by the three coauthors. In addition, the references of all articles were reviewed to identify any additional applicable publications that may have been missed. References from these articles were also obtained and citations are provided to readers with more details.

\section{Incidence and pathogenesis}

Globally, pancreatic exocrine cancer is the eighth leading cause of cancer-related death in men and the ninth in women [13]. In general, pancreatic cancer affects mostly individuals inhabiting the industrialized parts of the world [14,15]. Maoris in New Zealand, native Hawaiians, and Black American have the highest incidence of pancreatic cancer, while people living in India and Nigeria have the lowest $[14,15]$.

The disease is not often encountered prior the age of 45 and the incidence increases in older ages. Incidence varies and is greater in males (male-to-female ratio 1.3:1) and especially in black males ( 14.8 per 100,000 compared with 8.8 per 100,000 in the general population) [16].
In the pathogenesis of pancreatic exocrine cancer two pathways are implicated. One is that of acquired and/ or environmental risk factors and the other one is that of molecular carcinogenesis.

\section{Molecular pathogenesis of pancreatic cancer}

Many genetic mutations have been associated with pancreatic adenocarcinomas (Table 2). These can be categorized into five broad categories:

1. Mutational activation of oncogenes such as KRAS

2. Inactivation of tumor suppressor genes such as TP53, p16/CDKN2A, and SMAD4

3. Inactivation of genome maintenance genes, such as $h M L H 1$ and $\mathrm{MSH} 2$, which control the repair of DNA damage

4. Infrequent mutations

5. Genetic variants responsible for susceptibility pancreatic cancer.

Although most of these genetic aberrations represent somatic mutations, others are present in the germline of kindreds that carry a familial predisposition to pancreatic cancer [17]. 
Table 2 Genes involved in the pathogenesis of pancreatic cancer

\begin{tabular}{|c|c|c|}
\hline Targeted gene & Alteration prevalence & Altered gene function \\
\hline KRAS & $90 \%$ & Cell cycle activation (MAPK and PIK3CA pathway) \\
\hline P16/CDKN2A & $95 \%$ & CDK4 and CDK6 inhibition \\
\hline TP53 & $75 \%$ & Cellular stress response \\
\hline SMAD4 & $55 \%$ & Loss of TGF- $\beta$ induced tumor suppression \\
\hline KRAS & $80 \%$ & Cell cycle activation (MAPK and PIK3CA pathway) \\
\hline $\mathrm{P} 16 / \mathrm{CDKN} 2 \mathrm{~A}$ & Present only in high-grade & CDK4 and CDK6 inhibition \\
\hline TP53 & dysplasia and carcinoma & Cellular stress response \\
\hline SMAD4 & & Loss of TGF- $\beta$ induced tumor suppression \\
\hline GNAS & $60 \%-65 \%$ & Uncontrolled growth signaling \\
\hline RNF43 & $75 \%$ & Wnt signaling regulation \\
\hline KRAS & $30 \%-80 \%$ & Cell cycle activation \\
\hline RNF43 & $40 \%$ & Wnt signaling regulation \\
\hline P16/CDK2NA, TP53, SMAD4 & Only in high-grade tumors & \\
\hline CTNNB1 & $95 \%$ & Wnt $/ \beta$-catenin signaling pathway activation \\
\hline MEN1 & $45 \%$ & \\
\hline DAXX and ATRX & $45 \%$ & Chromatin remodeling \\
\hline PIK3CA, PTENand TSC2 & $14 \%$ & mTOR pathway \\
\hline VHL & $25 \%$ & HIF-1 $\alpha$ pathway \\
\hline APC- $\beta$-catenin & $25 \%$ & Cell signaling and adhesion \\
\hline KRAS & Rare & Cell cycle activation \\
\hline TP53 & Rare & Cellular stress response \\
\hline Fanconi anemia pathway genes & $45 \%$ & DNA repair mechanism \\
\hline CTNNB1 & $5 \%$ & Cell signaling and adhesion \\
\hline APC- $\beta$-catenin & $86 \%$ & Cell signaling and adhesion \\
\hline CTNNB1 & $55 \%$ & Cell signaling and adhesion \\
\hline $17 \mathrm{q} 25.1,7 \mathrm{p} 13,3 \mathrm{q} 29$ & Rare & Increased risk for pancreatic cancer \\
\hline TERT locus & Rare & Increased risk for pancreatic cancer \\
\hline rs6971499, rs7190458, rs9581943, rs16986825 & Rare & Increased risk for pancreatic cancer \\
\hline
\end{tabular}

\section{Mutational activation of oncogenes}

KRAS gene, located on chromosome $12 \mathrm{p}$, is one of the most frequently mutated genes in pancreatic cancer. This gene is the human homolog of a transforming gene isolated from the Kirsten rat sarcoma virus, hence the name, KRAS. As noted earlier, KRAS is an oncogene. Mutations in this gene, the vast majority of which are at codon 12 , are activating, leading to activation of the protein product of the gene. Over $90 \%$ of pancreatic cancers harbor a KRAS gene mutation $[18,19]$.

\section{Inactivation of tumor suppressor genes}

Loss of function of several tumor suppressor genes has been documented in pancreatic carcinomas. To abrogate gene function, both copies of the gene need to be inactivated.
Tumor suppressor genes that are inactivated in almost half cases of pancreatic exocrine cancer are p16/CDKN2A, TP53, and SMAD4 [20].

p16/CDKN2A gene on chromosome $9 \mathrm{p}$ is somatically inactivated in $95 \%$ of pancreatic cancers [21]. Loss of gene function abrogates an important control of the cell cycle in these tumors. Inherited mutations in the $p 16 / C D K N 2 A$ gene are one of the causes of the Familial Atypical Multiple Mole Melanoma (FAMMM) syndrome. Patients with the FAMMM syndrome have an increased risk of developing melanoma and a 20-34-fold increased risk of developing pancreatic cancer. The homozygous deletions that inactivate the $p 16 / C D K N 2 A$ gene frequently also inactivate an adjacent gene, MTAP [22,23].

TP53 gene, located on chromosome 17p, is a frequently targeted gene in human cancer. TP53 gene is inactivated in $75-85 \%$ of pancreatic cancers by an intragenic mutation coupled with loss of the second allele. Genetic inactivation 
of TP53 abrogates two important cell functions: regulation of cellular proliferation and cell death (apoptosis) in response to DNA damage [24].

SMAD-4 is a gene on chromosome 18, altered in 55\% of pancreatic neoplasms. The protein-product of SMAD4 gene functions in the transmission of intracellular signals, from transforming growth factor (TGF)- $\beta$ receptors within the cell membrane to the nucleus. When this gene is mutated, this function is inhibited and there is a loss of the TGF- $\beta$ induced tumor supression [25].

$B R C A 2$ gene on chromosome $13 \mathrm{q}$ is inactivated in fewer than $10 \%$ of pancreatic cancers. It is known that BRCA1/2 genes are involved in DNA repair and are implicated in breast and ovarian cancers, as well. Germline mutations in BRCA2 are associated with an increased risk of pancreatic cancer. This constitutes one of the most important causes of familial aggregation of pancreatic cancer. BRCA2 mutations are found in up to $17 \%$ of patients with familial pancreatic cancer [26].

PALB2 gene on chromosome 16p encodes for a BRCA2binding protein. Germline mutations in PALB2 are known to increase the risk of breast cancer, and germline truncating mutations in PALB2 have been identified in approximately $3 \%$ of individuals with familial pancreatic cancer [27].

Somatic STK11 mutations have been observed in $4 \%$ of pancreatic cancers, particularly those that arise in association with an intraductal papillary mucinous neoplasm. It is located on chromosome $19 \mathrm{p}$ and encodes for a serine/threonine kinase, which regulates cell polarity and functions as a tumor suppressor gene. Inactivation of the STK11 gene appears to play a role in both hereditary and sporadic pancreatic cancers [28].

Ataxia-telangiectasia-mutated (ATM) gene on chromosome $11 \mathrm{q}$ encodes for a member of the PI3/PI4-kinase family. ATM gene kinase product plays an important role in the cell response to DNA damage. Germline mutations in the ATM gene have been reported in $3 \%$ of families with familial pancreatic cancer, and somatic (acquired) ATM mutations have been reported in ductal adenocarcinomas [29].

\section{Inactivation of genome maintenance genes}

DNA mismatch repair genes such as $h M L H 1$ and $h M S H 2$ are well known for their important role in the pathogenesis of colorectal cancer, particularly in Lynch syndrome. Patients with this syndrome have germline mutations in one of several DNA mismatch repair genes and an elevated risk of several gastrointestinal cancers, including pancreatic cancer. These genes are mutated in approximately $4 \%$ of pancreatic cancers. Because of their distinctive "medullary" histologic appearance, pancreatic cancers with defects in DNA mismatch repair gene can sometimes be recognized histologically at the diagnostic microscope [30].

\section{Infrequent mutations}

A number of other genes have been identified that are only rarely mutated in pancreatic cancer; they are generally less important than KRAS, p16/CDKN2A, TP53, and SMAD4. Among the genes less often mutated are the following: EP300, SMARCA4, CDH1, EPHA3, FBXW7, MLL3, ROBO2, DPP6, BAI3, GPR133, GUCY1A2, PRKCG, ARID1A, and TGF- $\beta$-R2 [31].

\section{Genetic variants responsible for susceptibility pancreatic cancer}

High-penetrance mutations in BRCA1, BRCA2, CDKN2A, STK11, PALB2 and ATM along with mismatch repair genes and low-penetrance loci are associated with increased risk for pancreatic exocrine cancer. 9,925 pancreatic cancer cases and 11,569 controls, including 4,164 newly genotyped cases and 3,792 controls from North America, Central Europe and Australia were identified by Childs et al and a genome-wide association analysis was performed [32]. The result was the identification of three regions (17q25.1, 7p13, 3q29) associated significantly with increased risk for pancreatic cancer. A significant association at 2p13.3 was detected, a region known for its suggestive evidence in Han Chinese previously reported associations at $A B O$, telomerase reverse transcriptase (TERT), CLPTM1, KLF5, ZNRF3,BCAR1, NR5A2, PDX1 and LINC-PINT were reproduced. This study identified new loci associated with increased risk for the manifestation of pancreatic cancer.

A small number of common susceptibility loci have been identified for pancreatic cancer, one of which is marked by $r s 401681$ in the TERT-CLPTM1L gene region on chromosome 5p15.33. Campa et al [33] explored the notion whether additional single nucleotide polymorphisms (SNPs), independently of rs401681, could be associated to increased risk of pancreatic cancer. An in-depth analysis of genetic variability the telomerase RNA component (TERC) genes and TERT in 5,550 subjects with pancreatic adenocarcinoma and 7,585 controls from the PANcreatic Disease ReseArch (PANDoRA) and the PanScan consortia was performed. A statistically significant association was found between a variant in TERT and increased risk for pancreatic adenocarcinona [odds ratio: 0.85; 95\% confidence interval (CI), 0.80-0.90, $\mathrm{P}=8.3 \times 10^{-8}$ ]. Additional analysis adjusting $r s 2853677$ for $r \$ 401681$ showed that the two SNPs were independently associated with increased risk for pancreatic adenocarcinoma, as suggested by the low linkage disequilibrium between them [ $r(2): 0.07$, $\left.D^{\prime}: 0.28\right]$. In conclusion, this study proved that the TERT locus was associated with increased pancreatic cancer risk.

More recently, a multistage genome-wide association study [34] including 7,683 individuals with pancreatic adenocarcinoma and 14,397 controls was performed. Four new loci reached genome-wide statistical significance: $r s 16986825$ at $22 q 12.1, r s 9581943$ at $13 q 12.2, r s 6971499$ at $7 q 32.3$ and $r s 7190458$ at $16 q 23.1$. An independent signal in exon 2 of TERT at the established region $5 p 15.33(r s 2736098, \mathrm{OR}=0.80,95 \% \mathrm{CI}$ 0.76-0.85, $\mathrm{P}=9.8 \times 10^{-14}$ ) was identified along with a locus at 8q24.21 ( $r$ 1561927, $\mathrm{P}=1.3 \times 10^{-7}$ ) approaching genome-wide statistical significance located $455 \mathrm{~kb}$ telomeric of PVT1. This study resulted in the identification of multiple new susceptibility alleles for pancreatic adenocarcinoma worthy of exploration by future studies. 


\section{Treatment and outcome: an open challenge}

Various treatment strategies exist in dealing with pancreatic cancer, that vary according to institution and country. One could identify roughly three major categories of patients. The first category refers to patients who had a resection and are fit to receive adjuvant therapy. In Europe most of them will receive adjuvant treatment with gemcitabine [2], while in the United States it is common to apply protocols incorporating chemotherapy with radiation therapy [35-37]. Unfortunately, pancreatic cancer has an extremely high rate of systemic recurrence $(>80 \%)$ and of local recurrence $(>20 \%)$, with the majority occurring within two years [38]. The second category includes patients with locally advanced disease and a median survival of 1 year. Whether inoperable, borderline inoperable or medically inoperable, the protocols applied are those of the metastatic setting or chemoradiation rendering them operable or not. The third and most frequent category comprises patients who have or developed metastatic disease with a median survival of 6 months. Historical therapies such as gemcitabine monotherapy [2] or combinations of gemcitabine with other agents could achieve at best better symptom control and quality of life and offered nothing in terms of overall survival. Even erlotinib, when added to gemcitabine, increased the overall survival by two weeks only [4]. Despite the progress, in the light of the new standard of care that emerged a few years ago with the use of FOLFIRINOX [12], achieving a median survival of eleven months, and gemcitabine with nabpaclitaxel [8], achieving a median survival of eight months, the outcome in general remains poor, especially for those who proceed to receive further lines of therapy. The vast majority of patients will have deceased at two years after diagnosis.

For such a lethal and not rare disease, there is urgent need to identify prognostic and predictive markers. Although it is still early, a growing body of evidence suggests that individualized therapies in pancreatic cancer, based on the specific genetic alterations mentioned previously, will soon be a reality.

\section{Genetic factors affecting outcome}

Some genetic factors have been identified that influence the outcome of patients with pancreatic adenocarcinoma (Table 1). For the purpose of a better approach we present them as those that influence the outcome in the adjuvant and in the nonadjuvant setting.

\section{Post resection}

CONKO-001 trial [2] set the standard of care in the management of pancreatic adenocarcinoma following resection, which is the use of adjuvant chemotherapy with gemcitabine monotherapy. As mentioned, a large number of patients will ultimately recur with metastatic disease despite optimal management. Functional markers, currently being studied as indicators of disease progression, include stromal changes, microvascular density and tumor metabolism.

Pancreatic adenocarcinomas with loss of SMAD4 expression have a more dismal course with higher rates of distant metastases and poor prognosis. Blackford et al [39] conducted a study sequencing more than 750 million base pairs of DNA from 23,219 transcripts in a series of 24 adenocarcinomas of the pancreas. Thirty-nine genes mutated in more than one of these 24 cancers were sequenced in a separate panel of 90 well-characterized adenocarcinomas of the pancreas. Of these 114 patients, 89 underwent pancreatico-duodenectomy, and the somatic mutations in these cancers were correlated with the patient outcome.

SMAD4 gene inactivation was significantly associated with shorter overall survival (OS) (hazard ratio, 1.92; 95\%CI, 1.20-3.05; $\mathrm{P}=0.006$ ) adjusted for tumor size, margin status, lymph node status and age in multivariate analysis. Patients harboring SMAD4 gene inactivation had a median OS of 11.5 months, compared with 14.2 months for patients not harboring this inactivation, while mutations in TP53, CDKN2A, and presence of four or more mutations or homozygous deletions among the 39 most frequently mutated genes, did not have any impact on the survival. The conclusion was that SMAD4 gene inactivation was associated with a poorer outcome in patients with resected pancreatic adenocarcinoma. These data suggest that SMAD4 gene status may someday be useful for prognostic stratification and therapeutic decisionmaking, although this concept has to be further validated by larger studies.

Another molecular candidate under heavy investigation regarding tumor progression is CXC chemokine receptor type 4 (CXCR4). CXCR4 is a protein that in humans is encoded by the CXCR4 gene [40]. While CXCR4's expression is normally low or absent in healthy tissues, it is present in cancer cell lines. Expression of this receptor in cancer cells is associated with metastatic potential mainly in tissues containing a high concentration of CXCL12, such as bone marrow, lungs and liver [41]. For pancreatic adenocarcinoma, in a study performed by $\mathrm{Ma}$ et al [42], it was discovered that CXCR4 along glycogen synthase kinase- $3 \beta$ (GSK3 $\beta$ ) promote its proliferation and invasion potential. Inhibition of CXCR4 suppresses GSK3 $\beta$ expression, but the molecular mechanism by which CXCR4 promotes pancreatic adenocarcinoma is not well clarified yet. Therefore, they analyzed the effect of CXCR4 on GSK3 $\beta$ expression and the molecular impact on that. It was discovered that overexpression of CXCR4 promoted GSK3 $\beta$ expression and silencing of CXCR 4 suppressed GSK3 $\beta$ expression. Overexpression of CXCR4 resulted in activation of cyclin D1, expression of p-Akt and inhibition of p21 expression, while silencing of CXCR4 had the exact opposite effect. CXCR4 promotes GSK3 $\beta$ expression and PANC-1 invasion by Akt signaling and upregulates GSK3 $\beta$ expression, at least in part, at the level of transcription.

Human equilibrative nucleoside transporter 1 (hENT-1) is a biomarker and its function is allowing the passage, intracellulary and extracellulary, of pyrimidine nucleosides, like 5-fluorouracil (5-FU), capecitabine, and gemcitabine [43]. Microarrays from 434 patients in the ESPAC-3 trial [44] 
randomized to chemotherapy were stained. Afterwards, they were classified as having high and low hENT-1 expression and these expressing groups were compared. Median OS for gemcitabine-treated patients $(n=176)$ was 23.4 months (95\%CI, 18.3-26.0). Median OS for 176 patients treated with 5-FU/folinic acid was 23.5 (95\%CI, 19.8-27.3) months ( $\mathrm{P}=0.62)$. Median survival for patients in the low hENT1 expression group, treated with gemcitabine, was 17.1 (95\%CI, 14.3-23.8) months versus 26.2 (95\%CI, 21.2-31.4) months in the high hENT1 expression group $(\mathrm{P}=0.002)$. The level of hENT1 was not predictive of survival for the 28 patients of the observation group $(\mathrm{P}=0.54)$. Multivariate analysis confirmed the predictive value of hENT1 expression in gemcitabine-treated patients $(\mathrm{P}=0.003)$ [45]. One could argue that gemcitabine should be avoided in patients with low tumor hENT1 expression, but this needs validation by future and larger studies.

\section{Non-operable metastatic setting}

In the non-operable setting, there is a much greater need in tailored therapy, due to the higher risk for metastasis and lethal outcome. The most common drug used is gemcitabine and its combinations. It is a pyrimidine antimetabolite acting via inhibition of DNA polymerase and ribonucleotide reductase, cell cycle-specific for the S-phase of the cycle, thereby stopping DNA synthesis (also blocking cellular progression at G1/S-phase). Gemcitabine is phosphorylated intracellularly by deoxycytidine kinase to gemcitabine monophosphate, further phosphorylated to active metabolites gemcitabine diphosphate and gemcitabine triphosphate. Gemcitabine diphosphate inhibits DNA synthesis by inhibiting ribonucleotide reductase; gemcitabine triphosphate is incorporated into DNA and inhibits DNA polymerase [46].

Three major genes have been identified in the pharmacogenetics of pancreatic cancer regarding gemcitabine, hENT1, DCK, and CDA [47]. hENT-1 transports the drug across the cellular membrane in order to be phosphorylated and form active metabolites. hENT1 protein expression was strongly correlated with OS and disease-free survival (DFS) in patients with treated pancreatic cancer in the adjuvant setting [48]. Three mutations have been identified in the upstream of the gene [49] and there is a hypothesis that patients with metastatic pancreatic cancer and low levels of hENT1 expression may not benefit from gemcitabine therapy. Poplin et al [50] showed in their study that hENT1 expression was not of predictive value regarding gemcitabine activity and efficacy. Patients were randomly assigned to gemcitabine or CO-101, after providing a metastasis sample for blinded hENT1 assessment. Of 367 patients enrolled, hENT1 status was measured in 358 patients and roughly $65 \%$ had low expression of $h E N T 1$. No difference in OS was found between treatments in the low $h E N T 1$ expression subgroup or overall, with hazard ratios of 0.994 (95\%CI, 0.746-1.326) and 1.072 (95\%CI, 0.856-1.344), respectively. No major differences in toxicities between the treatment arms were observed. In the subgroup of gemcitabine-treated patients, no difference in survival between the high and low hENT1 expression subgroups was observed (hazard ratio: 1.147; 95\%CI, 0.809-1.626).

DCK phosphorylates gemcitabine to its active form, and AG genotype of a9846g was more sensitive to gemcitabine than GC genotype [60]. Higher levels increased survival, with a median OS of 21.7 months, in contrast to 14.6 months when low levels of DCK existed [51].

$C D A$ was evaluated in a study [52] and it was found that that patients with homozygous $C D A^{*} 3$ had extremely low activity, resulting in higher toxicity in terms of bone marrow suppression, pulmonary pneumonitis, hepatotoxicity, and capillary leak syndrome as $C D A$ metabolizes gemcitabine to its inactive renally excreted form.

Another active compound used in earlier times solo and now in combinations is 5-FU. DPD is the key enzyme responsible for metabolizing 5-FU. A study showing 68 stage II or higher pancreatic cancer samples investigated the correlation between DPD expression, 5-FU liver perfusion chemotherapy, and OS. Postoperative 5-FU-based therapy had a survival benefit in patients with low DPD levels [53].

As we pointed out earlier, DNA mismatch repair genes are mutated in approximately $4 \%$ of pancreatic cancers. Pancreatic cancers with microsatellite instability (MSI) may also be less responsive to chemotherapeutic agents such as 5-FU [54], as are colon cancers, at least in the adjuvant setting and appear to have a somewhat better prognosis than standard ductal adenocarcinomas, as it was demonstrated in the study by Nakata et al [55]. Forty-six histologically confirmed pancreatic adenocarcinomas in surgically resected patients were studied. The number of MSI-positive patients was eight, as determined by assessment of microsatellite variations in three or more of the eight tested markers. Univariate analysis was performed revealing that patients with MSI-positive tumors had significantly longer survival than those that were MSI-negative (median survival, 62 months versus 10 months, respectively; $\mathrm{P}=0.011$ ). Multivariate survival analysis validated the predictive value of the MSI status (hazard ratio: $5.577 ; \mathrm{P}=0.007$ ). The tumor-infiltrating leukocyte intensity in MSI-positive tumors was stronger than that in MSI-negative tumors, indicating that these tumors provoke the immune system, thus resulting in antitumor immunity. Therefore, the better prognosis in these patients can be attributed to the induced immunoreactivity of the tumor.

A pro drug of 5-FU is capecitabine. It undergoes hydrolysis in the liver and tissues to its active form 5-FU. 5-FU is a fluorinated pyrimidine antimetabolite that inhibits thymidylate synthetase, blocking the methylation of deoxyuridylic acid to thymidylic acid, thus disrupting DNA and RNA synthesis. Fluorouracil appears to be phase specific for the G1 and $S$ phases of the cell cycle. In experimental models, it has been proven that low levels of thymidine synthetase (TS) expression lead to better response rates to 5-FU and capecitabine and better survival of colon and liver cancer patients. However, additional experiments have merely stated that levels of TS may be associated with the disease stage, cell proliferation, and tumor differentiation. On the other hand, that does not mean that low levels indicate a better success in these patients. In conclusion, expression levels of TS mRNA may be a helpful 
predictive marker of the malignant potential of certain cancerous cells, thus guiding us in optimal cancer treatment targets and yielding higher survival rates among pancreatic cancer patients [56].

DPD is the first rate-limiting enzyme that 5-FU. Thymidine phosphorylase (TP) catalyzes the last step that converts capecitabine into 5-FU. TP/DPD ratio seems to correlate positively with the efficacy of capecitabine in human xenograft models. Saif et al demonstrated in a study that a lower TP/ DPD was associated with a survival benefit of 4 months [57]. This looks small, but we must take into account that the 5 -year survival is less than 5\%. TP/DPD ratio may be used as an independent marker for prognostication for locally advanced pancreatic cancer and it may help in determining the chemotherapy duration, choices and possibly toxicities as well. Larger studies are needed to study the relationship between TP/DPD ratio with these efficacy parameters.

Oxaliplatin, a platinum derivative, is an alkylating agent. Following intracellular hydrolysis, the platinum compound binds to DNA forming cross-links, which inhibit DNA replication and transcription, resulting in cell death with a nonspecific cell-cycle cytotoxicity. It used in the treatment of pancreatic adenocarcinoma in various combinations either with gemcitabine or in the FOLFIRINOX regimen. It has been reported [58] that the combination of gemcitabine and a platinum compound such as cisplatin is based on gemcitabineinduced increased formation and retention of DNA platinum adducts, which can be explained by a decrease in excision repair cross complementing group-1 (ERCC1)-mediated repair. Overall survival and response rates are prolonged when ERCC1 has a low protein or mRNA expression. A meta- analysis of 12 studies [58] with 836 patients with non-small cell lung cancer clearly demonstrated that low levels of ERCC1 mRNA or protein expression were associated with longer survival and a superior major response. Several other proteins are involved in the recognition of DNA damage, unwinding, subsequent excision of the damaged nucleotides and insertion of new deoxynucleoside triphosphates in the DNA, such as THFIIH, ERCC1/XPF, DNA polymerases $\delta$ and $\varepsilon$, and ligase 1 . Extrapolating in patients with pancreatic cancer, SNPs in any of these genes may affect the repair capacity and contribute to individual variations in chemotherapy response especially with platinum compounds [58].

Irinotecan [59] is a drug used mostly in later lines of treatment in the metastatic setting of pancreatic adenocarcinoma. In its active form, SN38 metabolite, is cleared by the biliary tract, after glucuronidation by uridine diphosphate-glucuronosyltransferase 1A1 (UGT1A1). UGT1A1 gene polymorphisms play a major part in the variability in the activity of UGT1A1. A review on the impact of the deficient $U G T 1 A 1^{*} 28$ variant on irinotecan efficacy and toxicity was produced by a French joint work group [60]. The conclusion was that for irinotecan doses at least equal to $180 \mathrm{mg} / \mathrm{m}^{2}$, patients homozygous for the UGT1A1 ${ }^{*} 28$ allele, are at increased risk of developing hematological and/or digestive toxicities and a dose reduction is warranted in homozygous $\star 28 /{ }^{*} 28$ patients.
Barriers to pancreatic tumor drug delivery include excess fibrous tissue and dense stroma mediated by molecules such as secreted protein acidic and rich in cysteine (SPARC). SPARC has multiple functions, such as cell adhesion, wound healing, signaling, angiogenetic inhibition, epithelial to mesenchymal transition, and action as a tumor suppressor gene [61]. It was found that high SPARC mRNA expression was a significantly independent prognostic marker for pancreatic cancer, with the five-year survival rate of patients with low SPARC mRNA level at $20 \%$ compared with $0 \%$ for patients with high SPARC mRNA level [62]. A previous study in mice [63] explored the role of SPARC in drug delivery. This study compared gemcitabine alone or with nab-paclitaxel. The nab-paclitaxel mouse group had increased intratumoral concentrations of gemcitabine and decreased peritumoral desmoplastic stroma concentration, thus indicating that nab-paclitaxel may target SPARC in the stroma allowing and facilitating the delivery of chemotherapy to the targeted tumor [63].

Human epidermal growth factor receptor type 1 (HER1/ EGFR) is overexpressed in most pancreatic adenocarcinomas and is strongly associated with a poorer outcome, metastatic potential and a more dismal prognosis overall [64-67]. Blocking HER1/EGFR tyrosine kinase signaling pathway has a negative effect regarding the growth rate and metastatic potential of human pancreatic tumor xenografts also improving the anticancer efficacy of gemcitabine. Erlotinib is an oral HER1/ EGFR tyrosine kinase inhibitor. In the trial of Moore et al [4], although a statistical significant survival of two weeks was achieved, EGFR status was not associated with response or disease stability. If we study with an open mind the results, it seems that there is a subgroup of patients who benefit from the addition of erlotinib, but we just do not have as yet the necessary biomarkers to target them.

Regarding targeting HER2, the results of the THERAPY trial are promising [68]. This was a phase 1-2 trial single-arm, non-randomized, multicenter trial, with weekly cetuximab administration $\left(400 \mathrm{mg} / \mathrm{m}^{2}\right.$, then $\left.250 \mathrm{mg} / \mathrm{m}^{2}\right)$. Then the patients were sequentially included in two trastuzumab dose levels: 3.0 or $4.0 \mathrm{mg} / \mathrm{kg}$, then 1.5 or $2.0 \mathrm{mg} / \mathrm{kg} /$ weekly. Endpoints were the objective response rate, safety, progressionfree survival (PFS) and OS. The rationale was to target simultaneously HER1/HER2. The higher dose level was defined as the trastuzumab recommended dose. During phase 2 ( $n=39$ patients), toxicities were in the vast majority asthenia and cutaneous reactions. No objective response was observed in any patient. Nine patients had stable disease but discontinued treatment due to toxicity. Median PFS was 1.8 months (95\%CI, 1.7-2.0 months) and median OS was 4.6 months (95\%CI, 2.7-6.6 months). Both were positively correlated with skin toxicity severity $(\mathrm{P}<0.05)$.

A more promising approach was exhibited by Kan et al [69]. The rationale was that while pancreatic adenocarcinomas widely express HER2, the level of expression is low. If HER2 expression in cancer cells could be enhanced by treatment with a given agent, then combination therapy with that agent and trastuzumab emtansine (T-DM1), a chemotherapeutic agent that is a conjugate of trastuzumab, might lead to significant antitumor effects against pancreatic 
adenocarcinomas. Five human pancreatic cancer cell lines were tested, including MIA PaCa-2. Three showed an increase in HER2 expression after gemcitabine treatment. The binding of T-DM1 to pretreated MIA PaCa-2 cells was higher than to untreated MIA PaCa- 2 cells. Treatment with gemcitabine and T-DM1 showed synergic cytotoxic effects on MIA PaCa- 2 cells in vitro. Cells in the G2M phase of the cell cycle were retained after gemcitabine treatment and showed higher levels of HER2 expression, possibly contributing to the synergic effect of and T-DM1. This needs further validation by larger trials.

We must also take into account that KRAS mutations, occurring in almost $90 \%$ of pancreatic cancers, constitute current anti-EGFR treatment ineffective as in colorectal cancer [70]. These mutations appear to occur very early in pancreatic carcinogenesis, as indicated by their presence in noninvasive precursors. KRAS gene mutations have been identified in noninvasive intraductal papillary mucinous neoplasms, in pancreatic intraepithelial neoplasia, and in noninvasive mucinous cystic neoplasms (and the prevalence of mutations increases with increasing degrees of dysplasia in these noninvasive precursor lesions [71]. Mouse studies provide compelling evidence that oncogenic KRAS is required for the formation for the initiation and maintenance of invasive pancreatic cancers [72]. Since mutations are both common and early events in pancreatic neoplasia, KRAS gene is an attractive target for the development of an early detection test.

BRCA2 mutations are found in up to $17 \%$ of patients with familial pancreatic cancer. The protein product of the BRCA2 gene plays an important role in the repair of DNA cross-linking damage. It is located on chromosome $13 \mathrm{q}$ and is inactivated in fewer than $10 \%$ of pancreatic cancers. The gene is almost always inactivated by a germline (inherited) mutation coupled with somatic loss of the second allele [73]. It has been suggested that this function of BRCA2 can be exploited therapeutically. In vitro studies suggest that pancreatic cancers with genetically inactivated BRCA2 are significantly more susceptible to DNA cross-linking agents, than pancreatic cancers with a genetically intact BRCA2. Indeed, several reports have documented remarkable therapeutic responses to DNA cross-linking agents such as mitomycin, cisplatin, or to poly ADP-ribose polymerase (PARP) inhibitors in patients whose cancers have inactivated BRCA2 [74,75].

Shirmer et al [76] conducted a study of 381 patients with pancreatic adenocarcinoma treated with gemcitabinebased therapy in order to identify the relationship between germline polymorphisms and overall survival. The A allele (26\% in Caucasians) at SNP rs11644322 in the putative tumor suppressor gene WWOX conferred worse prognosis. Median overall survival was 14 months (95\%CI, 12-15 months), 13 months (95\%CI, 11-15 months), and nine months (95\%CI, 7-12 months) for the GG, GA, and AA genotypes, respectively $(\mathrm{P}<0.001$ for trend in univariate log-rank assuming a codominant mode of inheritance; advanced disease subgroup P trend $<0.001)$. Mean overall survival was 25 months $(95 \% \mathrm{CI}$, 21-29 months), 19 months (95\%CI, 15-22 months), and 13 months (95\%CI, 10-16 months), respectively. This effect was held true after adjustment for age, performance status, stage, grading, and resection status and was comparable with the strongest established prognostic factors in multivariate analysis. Consistently, reduced responsiveness to gemcitabine, but not 5-FU, along with lower WWOX expression was demonstrated in lymphoblastoid cell linesharboring the AA genotype. Likewise, RNAi-mediated WWOX knockdown in pancreatic cancer cells confirmed differential cytostatic drug sensitivity. They concluded that decreased WWOX expression may interfere with gemcitabine sensitivity, and allele-specific binding at rs11644332 might be a causative molecular mechanism behind the observed clinical associations.

\section{Discussion}

We are entering a new era where individualized therapy will play a major role. Genomic sequencing has a great potential to improve diagnosis, provide information about the tumor and help guide the choice of a more tailor-made therapy. In this direction there are a few challenges ahead.

Tissue sampling is a necessity for the appropriate genomics test and we must take into account that the intrinsic biology of the tumor is evolving after each therapy, so it is a question when and how often during the course of the treatment a sample must be obtained. Also, all these methods of genomic sequencing need to be further validated in the context of clinical trials and be further explored in real patients in conjunction with standard treatments, in order to export safe and useful conclusions. Last but not least, in the light of the rapid rising costs in modern medicine, there is a need for screening to avoid the high prevalence of metastatic pancreatic cancer, which is inoperable and incurable at the time and also assessment of biomarkers so that we could choose those patients who would benefit the most.

Tailor-made personalized medicine in pancreatic cancer, as in oncology in general, is the way of the future. In the evolving field of circulating tumor cells (CTC) progress is being made. In a pilot study of Earl et al [77] the aim was to assess tumorspecific DNA detection in plasma and CTC detection as prognostic markers in pancreatic cancer. KRAS mutant cellular free DNA (cfDNA) was detected in $26 \%$ of patients of all stages and this correlated strongly with overall survival, that is 60 days (95\%CI, 19-317) for KRAS mutation positive versus 772 days for KRAS mutation negative (95\%CI, 416-1127). Tumorspecific cfDNA detection and CTC detection are promising markers for the management of patients, although there is a need to validate these results by a larger patient cohort.

As we stated earlier, there is an urgent need for novel and reliable biomarkers for the diagnosis and prognostication of pancreatic cancer. Circulating microRNAs (miRNAs) have been extensively profiled in blood samples, but few studies have performed adequate validation of candidate markers. $\mathrm{Xu}$ et al [78] investigated pre-operative plasma miRNAs from patients over three phases and three surgical centers. They revealed that miR-486-5p and miR-938 were able to discriminate pancreatic adenocarcinoma patients from healthy controls and those with chronic pancreatitis. The diagnostic ability of miR486-5p for identifying pancreatic adenocarcinoma patients 
from healthy controls was comparable to that of CA 19-9. This study provides further evidence for the use of blood-based miRNAs as diagnostic biomarkers in pancreatic cancer.

Next generation sequencing is an evolving field to determine potential molecularly guided therapy options for patients with pancreatic adenocarcinoma. A study was conducted by Wright et al [79] in thirty patients with resected pancreatic adenocarcinoma and next-generation sequencing was performed from paraffin-tumor blocks. The incidence of mutations were as follows: KRAS, 87\%; TP53, 63\%; CDKN2A, 20\%; SMAD4, 20\%; and EGFR, 7\%. Multiple mutations were found in $73 \%$. All CDKN2A mutations occurred in male patients $(\mathrm{P}=0.06)$, and there was a trend toward younger patient age in this group $(\mathrm{P}=0.13)$. Potential for the US Food and Drug Administration-approved targeted therapies was identified in 8 of 30 (27\%). In addition, 29 of 30 (97\%) had mutations applicable for ongoing phases I or II clinical trials.

In conclusion, it seems that the proper way to move forward is genomic sequencing in multiple and various tissue samples of the tumor, validation of the methods, proper selection of patients who could benefit with the emergence of potent prognostic and predictive biomarkers, CTCs and their role as biomarkers and more research in the context of clinical trials regarding immunotherapy.

\section{Concluding remarks}

As in other malignancies, such as non-small cell lung cancer and colorectal cancer, where genomic profiling of the tumor affects prognosis and treatment selection, it seems that this could also be the case in pancreatic cancer. We have already made a remarkable progress in translational research and it is time to apply the knowledge we have acquired in clinical practice. Molecular pathogenesis of pancreatic cancer nowadays is well understood as are genomic, epigenetic and proteomic factors affecting outcome after therapy. An upgrade in translational research is much needed and translating these findings into clinical trials and new standard of care is of high importance. Whether in the adjuvant setting or not, thankfully, there is no paucity of tissue and therefore molecular profiling is feasible. Since we have the knowledge and the tools, every effort should be targeted towards the goal of obtaining tissue, profiling the tumor accordingly, identifying the aforementioned factors influencing the outcome, and delivering the appropriate treatment.

\section{References}

1. Siegel RL, Miller KD, Jemal A. Cancer statistics, 2015. CA Cancer J Clin 2015;65:5.

2. Oettle H, Post $\mathrm{S}$, Neuhaus $\mathrm{P}$, et al. Adjuvant chemotherapy with gemcitabine vs observation in patients undergoing curative-intent resection of pancreatic cancer: a randomized controlled trial. JAMA 2007;297:267-277.
3. Burris HA $3^{\text {rd }}$, Moore MJ, Andersen J, et al. Improvements in survival and clinical benefit with gemcitabine as first-line therapy for patients with advanced pancreas cancer: a randomized trial. J Clin Oncol 1997;15:2403-2413.

4. Moore MJ, Goldstein D, Hamm J, et al. National Cancer Institute of Canada Clinical Trials Group. Erlotinib plus gemcitabine compared with gemcitabine alone in patients with advanced pancreatic cancer: a phase III trial of the National Cancer Institute of Canada Clinical Trials Group. J Clin Oncol 2007;25:1960-1966.

5. Louvet $\mathrm{C}$, Labianca R, Hammel P, et al. Gemcitabine in combination with oxaliplatin compared with gemcitabine alone in locally advanced or metastatic pancreatic cancer: results of a GERCOR and GISCAD phase III trial. J Clin Oncol 2005;23:3509-3516.

6. Heinemann V, Quietzsch D, Gieseler F, et al. Randomized phase III trial of gemcitabine plus cisplatin compared with gemcitabine alone in advanced pancreatic cancer. J Clin Oncol 2006;24:3946-3952.

7. Herrmann R, Bodoky G, Ruhstaller T, et al. Gemcitabine plus capecitabine compared with gemcitabine alone in advanced pancreatic cancer: a randomized, multicenter, phase III trial of the Swiss Group for Clinical Cancer Research and the Central European Cooperative Oncology Group. J Clin Oncol 2007;25:2212-2217.

8. Von Hoff DD, Ramanathan RK, Borad MJ, et al. Gemcitabine plus nab-paclitaxel is an active regimen in patients with advanced pancreatic cancer: a phase I/II trial. J Clin Oncol 2011;29:4548-4554.

9. Cartwright TH, Cohn A, Varkey JA, et al. Phase II study of oral capecitabine in patients with advanced or metastatic pancreatic cancer. J Clin Oncol 2002;20:160-164.

10. Xiong HQ, Varadhachary GR, Blais JC, Hess KR, Abbruzzese JL, Wolff RA. Phase 2 trial of oxaliplatin plus capecitabine (XELOX) as second-line therapy for patients with advanced pancreatic cancer. Cancer 2008;113:2046-2052.

11. Hosein PJ, de Lima Lopes G Jr, Pastorini VH, et al. A phase II trial of nab-paclitaxel as second-line therapy in patients with advanced pancreatic cancer. Am J Clin Oncol 2013;36:151-156.

12. Conroy T, Desseigne F, Ychou M, et al. FOLFIRINOX versus gemcitabine for metastatic pancreatic cancer. $N$ Engl $J$ Med 2011;364:1817-1825.

13. Jemal A, Bray F, Center MM, Ferlay J, Ward E, Forman D. Global cancer statistics. CA Cancer J Clin 2011;61:69-90.

14. Boyle P, Hsieh CC, Maisonneuve P, La Vecchia C, Macfarlane GJ, Walker AM. Epidemiology of pancreas cancer (1988). Int J Pancreatol 1989;5:327-346.

15. Hariharan D, Saied A, Kocher HM. Analysis of mortality rates for pancreatic cancer across the world. HPB (Oxford) 2008;10:58-62.

16. Zhang J, Dhakal I, Ning B, Kesteloot H. Patterns and trends of pancreatic cancer mortality rates in Arkansas, 1969-2002: a comparison with the US population. Eur J Cancer Prev 2008;17:18-27.

17. Hruban RH, Petersen GM, Ha PK, Kern SE. Genetics of Pancreatic Cancer. From genes to families. Surg Oncol Clin N Am 1998;7:1-23.

18. Hruban RH, van Mansfeld A, Offerhaus JA, et al. K-ras oncogene activation in adenocarcinoma of the human pancreas. A study of 82 carcinomas using a combination of mutant-enriched polymerase chain reaction analysis and allele-specific oligonucleotide hybridization. Am J Pathol 1993;143:545-554.

19. Almoguera C, Shibata D, Forrester K, Martin J, Arnheim N, Perucho M. Most human carcinomas of the exocrine pancreas contain mutant c-K-ras genes. Cell 1988;53:549-554.

20. Rozenblum E, Schutte M, Goggins M, et al. Tumor-suppressive pathways in pancreatic carcinoma. Cancer Res 1997;57:1731-1734.

21. Caldas C, Hahn SA, da Costa LT, et al. Frequent somatic mutations and homozygous deletions of the p16 (MTS1) gene in pancreatic adenocarcinoma. Nat Genet 1994;8:27-32. 
22. Hustinx SR, Hruban RH, Leoni LM, et al. Homozygous deletion of the MTAP gene in invasive adenocarcinoma of the pancreas and in periampullary cancer: a potential new target for therapy. Cancer Biol Ther 2005;4:83-86.

23. Subhi AL, Tang B, Balsara BR, et al. Loss of methylthioadenosine phosphorylase and elevated ornithine decarboxylase is common in pancreatic cancer. Clin Cancer Res 2004;10:7290-7296.

24. Redston MS, Caldas C, Seymour AB, et al. p53 mutations in pancreatic carcinoma and evidence of common involvement of homocopolymer tracts in DNA microdeletions. Cancer Res 1994;54:3025-3033.

25. Derynck R, Zhang YE. Smad-dependent and Smad-independent pathways in TGF-beta family signalling. Nature 2003;425:577-584.

26. Murphy KM, Brune KA, Griffin C, et al. Evaluation of candidate genes MAP2K4, MADH4, ACVR1B, and BRCA2 in familial pancreatic cancer: deleterious BRCA2 mutations in $17 \%$. Cancer Res 2002;62:3789-3793.

27. Slater EP, Langer P, Niemczyk E, et al. PALB2 mutations in European familial pancreatic cancer families. Clin Genet 2010;78:490-494.

28. Su GH, Hruban RH, Bansal RK, et al. Germline and somatic mutations of the STK11/LKB1 Peutz-Jeghers gene in pancreatic and biliary cancers. Am J Pathol 1999;154:1835-1840.

29. Roberts NJ, Jiao Y, Yu J, et al. ATM mutations in patients with hereditary pancreatic cancer. Cancer Discov 2012;2:41-46.

30. Wilentz RE, Goggins $M$, Redston $M$, et al. Genetic, immunohistochemical, and clinical features of medullary carcinoma of the pancreas: A newly described and characterized entity. Am J Pathol 2000;156:1641-1651.

31. Waddell N, Pajic M, Patch AM, et al. Whole genomes redefine the mutational landscape of pancreatic cancer. Nature 2015;518:495-501.

32. Childs EJ, Mocci E, Campa D, et al. Common variation at 2p13.3, $3 q 29,7 \mathrm{p} 13$ and $17 \mathrm{q} 25.1$ associated with susceptibility to pancreatic cancer. Nat Genet 2015;47:911-916.

33. Campa D, Rizzato C, Stolzenberg-Solomon R, et al. TERT gene harbors multiple variants associated with pancreatic cancer susceptibility. Int J Cancer 2015;137:2175-2183.

34. Wolpin BM, Rizzato C, Kraft P, et al. Genome-wide association study identifies multiple susceptibility loci for pancreatic cancer. Nat Genet 2014;46:994-1000.

35. Neoptolemos JP, Stocken DD, Friess H, et al. A randomized trial of chemoradiotherapy and chemotherapy after resection of pancreatic cancer. N Engl J Med 2004;350:1200-1210.

36. Loehrer PJ Sr, Feng Y, Cardenes H, et al. Gemcitabine alone versus gemcitabine plus radiotherapy in patients with locally advanced pancreatic cancer: an Eastern Cooperative Oncology Group trial. J Clin Oncol 2011;29:4105-4112.

37. Regine WF, Winter KA, Abrams RA, et al. Fluorouracil vs gemcitabine chemotherapy before and after fluorouracil-based chemoradiation following resection of pancreatic adenocarcinoma: a randomized controlled trial. JAMA 2008;299:1019-1026.

38. Van den Broeck A, Sergeant G, Ectors N, Van Steenbergen W, Aerts R, Topal B. Patterns of recurrence after curative resection of pancreatic ductal adenocarcinoma. Eur J Surg Oncol 2009;35:600-604.

39. Blackford A, Serrano OK, Wolfgang CL, et al. SMAD4 gene mutations are associated with poor prognosis in pancreatic cancer. Clin Cancer Res 2009;15:4674-4679.

40. Moriuchi M, Moriuchi H, Turner W, Fauci AS. Cloning and analysis of the promoter region of CXCR4, a coreceptor for HIV-1 entry. J Immunol 1997;159:4322-4329.

41. Sun X, Cheng G, Hao M, et al. CXCL12/CXCR4/CXCR7 Chemokine axis and cancer progression. Cancer Metastasis Rev 2010;29:709-722.

42. Ma S, Li Q, Pan F. CXCR4 promotes GSK3 $\beta$ expression in pancreatic cancer cells via the Akt pathway. Int J Clin Oncol 2015;20:525-530.
43. Yao SY, Ng AM, Cass CE, Baldwin SA, Young JD. Nucleobase transport by human equilibrative nucleoside transporter 1 (hENT1). J Biol Chem 2011;286:32552-32562.

44. Neoptolemos JP, Stocken DD, Bassi C, et al. Adjuvant chemotherapy with fluorouracil plus folinic acid vs gemcitabine following pancreatic cancer resection: a randomized controlled trial. JAMA 2010;304:1073-1081.

45. Greenhalf W, Ghaneh P, Neoptolemos JP, et al. Pancreatic cancer hENT1 expression and survival from gemcitabine in patients from the ESPAC-3 trial. JNCI J Natl Cancer Inst 2014;106:1-10.

46. Heinemann V, Hertel LW, Grindey GB, Plunkett W. Comparison of the cellular pharmacokinetics and toxicity of 2'2'-difluorodeoxycytidine and 1-beta-Darabinofuranosylcytosine. Cancer Res 1988;48:4024-4031.

47. Frank TS, Sun X, Zhang Y, et al. Genomic profiling guides the choice of molecular targeted therapy of pancreatic cancer. Cancer Lett 2015;363:1-6.

48. Farrell JJ, Elsaleh H, Garcia M, et al. Humanequilibrative nucleoside transporter 1 levels predict response to gemcitabinein patients with pancreatic cancer. Gastroenterology 2009;136:187-195.

49. Myers SN, Goyal RK, Roy JD, Fairfull LD, Wilson JW, Ferrell RE. Functional single nucleotide polymorphism haplotypes in the human equilibrative nucleoside transporter 1, Pharmacogenet. Genomics 2006;16:315-320.

50. Poplin E, Wasan H, Rolfe L, et al. Randomized, multicenter, phase II study of CO-101 versus gemcitabine in patients with metastatic pancreatic ductal adenocarcinoma: including a prospective evaluation of the role of hENT1 in gemcitabine or CO-101 sensitivity. J Clin Oncol 2013;31:4453-4461.

51. Sebastiani V, Ricci F, Rubio-Viquiera B, et al. Immunohistochemical and genetic evaluation of deoxycytidine kinase in pancreatic cancer: relationship to molecular mechanisms of gemcitabine resistance and survival. Clin Cancer Res 2006;12:2492-2497.

52. Ueno H, Kaniwa N, Okusaka T, et al. Homozygous $\mathrm{CDA}^{\star} 3$ is a major cause of life-threatening toxicities in gemcitabine-treated Japanese cancer patients. Br J Cancer 2009;100:870-873.

53. Fischel JL, Etienne MC, Spector T, Formento P, Rente N, Milano G. Dihydropyrimidine dehydrogenase: a tumoral target for fluorouracil modulation. Clin Cancer Res 1995;1:991-996.

54. Ribic CM, Sargent DJ, Moore MJ, et al. Tumor microsatelliteinstability status as a predictor of benefit from fluorouracilbased adjuvant chemotherapy for colon cancer. $N$ Engl J Med 2003;349:247-257.

55. Nakata B, Wang YQ, Yashiro M, et al. Prognostic value of microsatellite instability in resectable pancreatic cancer. Clin Cancer Res 2002;8:2536-2540.

56. Peters GJ, Backus HH, Freemantle S, et al. Induction of thymidylate synthase as a 5-fluorouracil resistance mechanism. Biochim Biophys Acta 2002;1587:194-205.

57. Saif MW, Hashmi S, Bell D, Diasio RB. Prognostication of pancreatic adenocarcinoma by of expression thymidine phosphorylase/ dihydropyrimidine dehydrogenase ratio and its correlation with survival. Expert Opin Drug 2009;8:507-514.

58. Peters GJ, Avan A, Ruiz MG, et al. Predictive role of repair enzymes in the efficacy of cisplatin combinations in pancreatic and lung cancer. Anticancer Res 2014;34:435-442.

59. Wagener DJ, Verdonk HE, Dirix LY, et al. Phase II trial of CPT11 in patients with advanced pancreatic cancer, an EORTC early clinical trials group study. Ann Oncol 1995;6:129-132.

60. Etienne-Grimaldi MC, Boyer JC, Thomas F, et al. UGT1A1 genotype and irinotecan therapy: general review and implementation in routine practice. Fundam Clin Pharmacol 2015;29:219-237.

61. Chen S, Zhang J, Wang R Luo X, Chen H. The platinum treatments of advanced non small cell lung cancer, is low negative ERCC1 expression better than high positive ERCC1 expression? A meta 
analysis. Lung Cancer 2010;70:63-70.

62. Kupprion C, Motamed K, Sage EH. SPARC (BM-40, osteonectin) inhibits the mitogenic effect of vascular endothelial growth factor on microvascular endothelial cells. J Biol Chem 1998;273:2963529640.

63. Gao J, Song J, Huang H, et al. Methylation of the SPARC gene promoter and its clinical implication in pancreatic cancer. J Exp Clin Cancer Res 2010;29:28.

64. Von Hoff DD, Ramanathan RK, Borad MJ, et al. Gemcitabine plus nab-paclitaxel is an active regimen in patients with advanced pancreatic cancer: a phase I/II trial. J Clin Oncol 2011;29:45484554.

65. Fjällskog ML, Lejonklou MH, Oberg KE, et al. Expression of molecular targets for tyrosine kinase receptor antagonists in malignant endocrine pancreatic tumors. Clin Cancer Res 2003;9:1469-1473.

66. Tobita K, Kijima H, Dowaki S, et al. Epidermal growth factor receptor expression in human pancreatic cancer: Significance for liver metastasis. Int J Mol Med 2003;11:305-309.

67. Xiong HQ. Molecular targeting therapy for pancreatic cancer. Cancer Chemother Pharmacol 2004;54(Suppl 1):S69-S77.

68. Assenat E, Azria D, Mollevi C, et al. Dual targeting of HER1/ EGFR and HER2 with cetuximab and trastuzumab in patients with metastatic pancreatic cancer after gemcitabine failure: results of the "THERAPY" phase 1-2 trial. Oncotarget 2015;6:1279612808.

69. Kan S, Koido S, Okamoto M, et al. Up-regulation of HER2 by gemcitabine enhances the antitumor effect of combined gemcitabine and trastuzumab emtansine treatment on pancreatic ductal adenocarcinoma cells. BMC Cancer 2015;15:726.

70. Valtorta E, Misale S, Sartore-Bianchi A, et al. KRAS gene amplification in colorectal cancer and impact on response to EGFR-targeted therapy. Int J Cancer 2013;133:1259-1265.

71. Moskaluk CA, Hruban RH, Kern SE. p16 and K-ras gene mutations in the intraductal precursors of human pancreatic adenocarcinoma. Cancer Res 1997;57:2140-2143.

72. Aguirre AJ, Bardeesy N, Sinha M, et al. Activated Kras and Ink4a/ Arf deficiency cooperate to produce metastatic pancreatic ductal adenocarcinoma. Genes Dev 2003;17:3112-3126.

73. Hahn SA, Greenhalf B, Ellis I, et al. BRCA2 germline mutations in familial pancreatic carcinoma. J Natl Cancer Inst 2003;95:214-221.

74. Lowery MA, Kelsen DP, Stadler ZK, et al. An emerging entity: pancreatic adenocarcinoma associated with a known BRCA mutation: clinical descriptors, treatment implications, and future directions. Oncologist 2011;16:1392-1402.

75. Kaufman B, Shapira-Frommer R, Schmutzler RK, et al. Olaparib monotherapy in patients with advanced cancer and a germline BRCA1/2 mutation. J Clin Oncol 2015;33:244-250.

76. Schirmer MA, Lóske CM, Roppel S, et al. Relevance of Sp binding site polymorphism in WWOX for treatment outcome in pancreatic cancer. JNCI J Natl Cancer Inst 2016:108:1-11.

77. Earl J, Garcia-Nieto S, Martinez-Avila JC, et al. Circulating tumor cells $(\mathrm{Ctc})$ and kras mutant circulating free Dna (cfdna) detection in peripheral blood as biomarkers in patients diagnosed with exocrine pancreatic cancer. BMC Cancer 2015;15:797.

78. Le Large TY, Meijer LL, Mato Prado M, Kazemier G, Frampton AE, Giovannetti E. Circulating microRNAs as diagnostic biomarkers for pancreatic cancer. Expert Rev Mol Diagn 2015;15:1-5.

79. Wright GP, Chesla DW, Chung MH. Using next-generation sequencing to determine potential molecularly guided therapy options for patients with resectable pancreatic adenocarcinoma. Am J Surg 2016;211:506-511. 